\title{
Smart Car System Based on Electromagnetism Technology
}

\author{
Changhui $\mathrm{Hu}$ \\ College of Mechatronics and Control Engineering \\ Hubei Normal University \\ conanhu_gdnt@yahoo.cn
}

\begin{abstract}
Introduce Fressscale MC9S12XS256 as the core control unit of the self-tracing smart car system. Fast and accurate information extracting and processing is the guarantee of the sensitive smart car quickly moving along the track. To the smart car system based on electromagnetic technology, create one method of path position detection and its technology details. Tests show this method can be good at forward-looking, real-time and accuracy.
\end{abstract}

KEYWORDS-Electromagnetism technology, Smart car, Path position detection method

\section{INTRODUCTION}

The combination of intelligent technology and Automobile has been extensive attention in modern industry area, which will play an important role in future industry and daily life.

The smart car system is built based on electromagnetic sensor of LC resonant circuit and Freescale's 16-bit microcontroller MC9S12XS256. A wire with the AC (alternation current) range from $80 \mathrm{~mA}$ to $120 \mathrm{~mA}$ locates in the centre of the smart car's running path, the electromagnetic sensor detects the electromagnetic field strength produced by the wire on the path, and the detection signal determines the direction and speed for the smart car's running. The difficult of electromagnetic technology smart car system is the detection method of electromagnetic field signal and path identification algorithm. This paper proposes a solution of path position detection method. Tests show that the forward-looking, stability and sensitivity of this method are very good, and smart car can run fast and stable on its path.

\section{THE OVERALL STRUCTURE OF THE SMART CAR SYSTEM}

According to module function, electromagnetic technology smart car can be divided into seven parts as follows, minimum system module based on microcontroller MC9S12XS256, electromagnetic sensor module, power management module, speed feedback module, steering drive module, motor drive module and wireless transmission module. Figure.1 shows the overall structure of intelligent vehicle system plan.

In smart car system, MC9S12XS256 minimum system module integrated on-chip A / D converter(16-channel), PWM output module and so on, A/D converter is used to collect and convert the analog signals detected by electromagnetic sensor, and PWM output module is used to drive the steering and motor. The electrical technology of other parts of the smart car are very mature, and do not do introduce one by one.

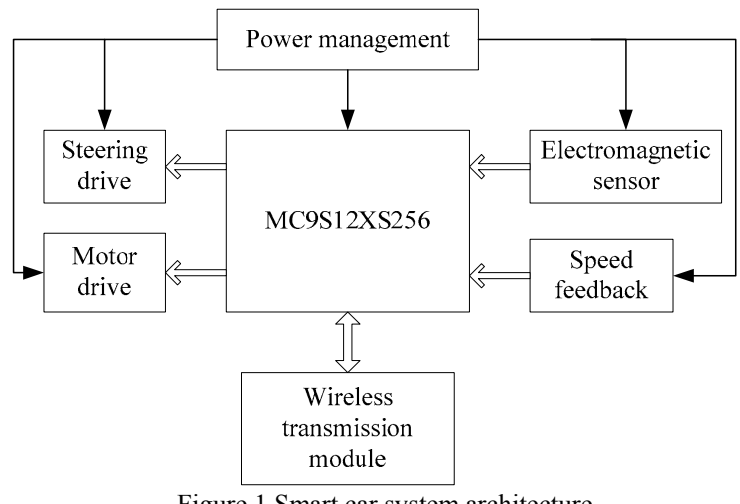

Figure.1 Smart car system architecture

\section{ANALYSIS OF RUNNING PATH}

A wire with the AC range from $80 \mathrm{~mA}$ to $120 \mathrm{~mA}$ locates in the centre of the running path of electromagnetic technology smart car. This wire gives the navigation information for the smart car. The frequency of the AC through the wire is $20 \mathrm{kHz}$, which belong to very low frequency (VLF) electromagnetic wave is much greater than the width of smart car. Figure. 2 shows the electromagnetic field distribution around the wire. The distribution of magnetic field around smart car is a serial of concentric circles, whose axis is the navigation wire; the magnetic field strength is equal on the same circle[1].

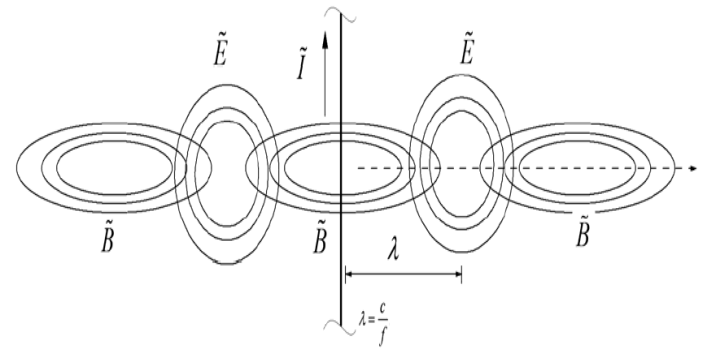

Figure 2. Electromagnetic field distribution around navigation wire

The relationship between electromotive force(EMF) of the induction coil and $\frac{\mathrm{h}}{\mathrm{x}^{2}+\mathrm{h}^{2}}$ is proportional, $\mathrm{h}$ is the 
vertical height between induction coil and navigation wire, $\mathrm{x}$ is the horizontal distance between them, the function relationship graph between EMF and $\mathrm{x}$ is shown in Figure.3[2].

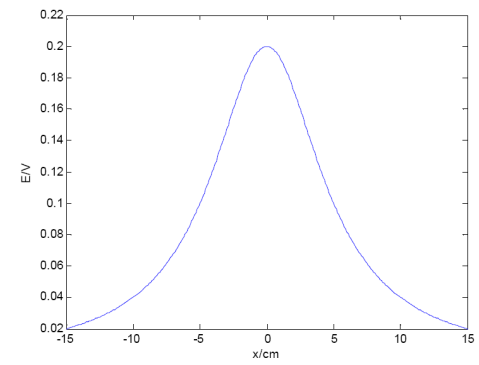

Figure 3. Function relationship graph between EMF and $\mathrm{x}$

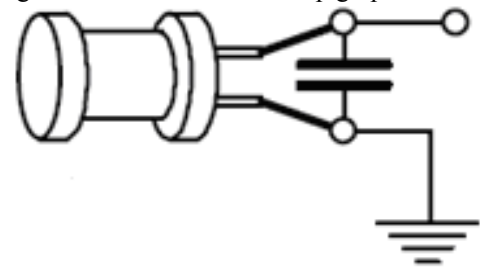

Figure 4. LC resonant circuit

\section{ELECTROMAGNETIC SENSOR}

Theoretical analysis and experimental testing show that $\mathrm{LC}$ resonant circuit can be used to detect the magnetic field signal of the navigation wire. LC resonant circuit is shown in Figure.4. According theoretical calculation, its inductance is $10 \mathrm{mH}$, and capacitance is $6.8 \mathrm{nF}$, and these two components can be bought from electronical market easily.

The LC resonant circuit is able to detect the magnetic field signals, and convert them to electrical signals, however the electrical signals are very weak, and can not be directly used as navigation signals for the controlling of smart car. Electrical signals must be amplified, and then can be used to control the smart car as navigation signals.

Operational amplifier circuit can effectively amplify the output signal of LC resonant circuit, as shown in Figure.5, electromagnetic sensor circuit diagram based on LC resonant and operational amplifier. To adjust resistor R102 can control the output signal of magnification of the Operational amplifier circuit magnification. This kind of electromagnetic sensor circuit can effectively avoid the impact of the unequal parameters of electromagnetic sensors circuit, which caused by the differences of the components themselves. Operational amplifier circuit has higher processing speed, better real-time than transistor amplifier circuit. Experiments show that the advantages the electromagnetic sensor based on Operational amplifier circuit are as follows, magnification is easy to control, Antielectromagnetic interference is good, Output signals change gently, and dynamic signals are stability. The EMF signal output of single electromagnetic sensor circuit is $20 \mathrm{kHz}$ sine wave as shown in Figure.6.

\section{INDUCTANCE COILS ARRANGEMENT OF PATH POSITION METHOD}

The inductance coils arrangement of electromagnetic sensor module of path position detection method was shown in Figure.7. Black circles represent the coils, all coils are in the same horizontal plane, 9 coils in front row, from left to right numbered 1 to 9,5 coils in rear row, from left to right number 1 to 5 . The distance between front row and left row is $12 \mathrm{~cm}$, the length of front row is $24 \mathrm{~cm}$, rear row is $12 \mathrm{~cm}$, and two neighbour coils is $3 \mathrm{~cm}$, two corresponding coils of 3 to 7 in front row and 1 to 5 in rear row are in the same axis paralleled the axis of the smart car .The whole electromagnetic sensor module installed in front of the smart car, and rear row near its front wheel, and the car body width is $15 \mathrm{~cm}$.

The principle of position detection method is, when electromagnetic sensor module located above the navigation wire, the inductance coil nearest to navigation wire produces maximum navigation signal in each row. Thus, in front row the inductance coil of maximum output signal could be selected.Use the same method the inductance coil could be selected in rear row.

When the smart car drives on straight navigation wire, the connection line of two selected coils in front row and rear row is parallel to the navigation wire, in this condition, do not adjust the smart car's driving direction. When the smart car drives on curved navigation wire, according to the angle between the connection line of two selected coils and the navigation wire, adjust the direction of smart car to cross curved path easily.

Position detected method detects the position of the navigation wire through the two coils of front row and rear row. This method has better forward-looking, and do not depend on the value of AC current through the navigation wire, can accurately distinguish straight path and curved path, and provide enough information for the running of the smart car stably and quickly.

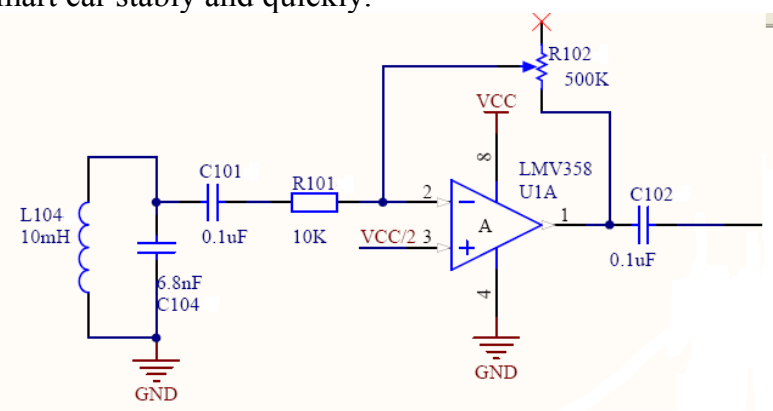

Figure 5. Single Electromagnetic sensor circuit

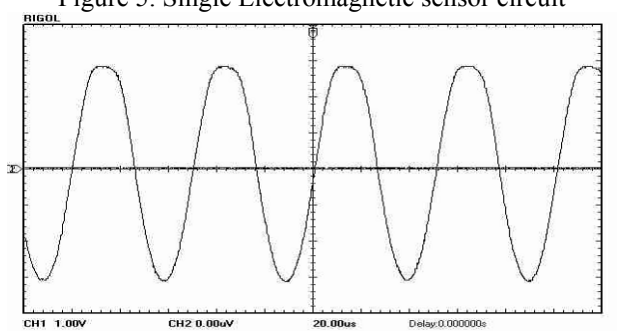


Figure 6. Output signal of single electromagnetic sensor circuit

\section{ALGORITHM OF PATH POSITION DETECTION METHOD}

As electromagnetic sensor module of path position detection method contain 14 coils, collecting and calculating the output signals of all coils may cost much time. According to the principle of path position detection method, only one coil of maximum output signal (nearest coil to navigation wire) has to be selected in front row, so does in rear row. The search method of maximum output signal coil is to search from middle to right and left sides to find the nearest coil to navigation wire in each row. First collect signals of coil 4,5 and 6 in front row, if the output signal of coil 4 is greater than that of coil 5 . collect the output signals of coil 3 and 2 , if the output signal of coil 2 is greater than that of coil 3 , collect the output signals of coil 1 . Once finding the maximum output signal coil, then give up the search. For instance, shown in figure 10, if the output signal of coil 3 (red circle) is greater than that of coil 4 and coil 2 , so coil 3 is the coil of maximum output signal in front row, search done, the output signals of coil $1,7,8$ and 9 have not be collected.

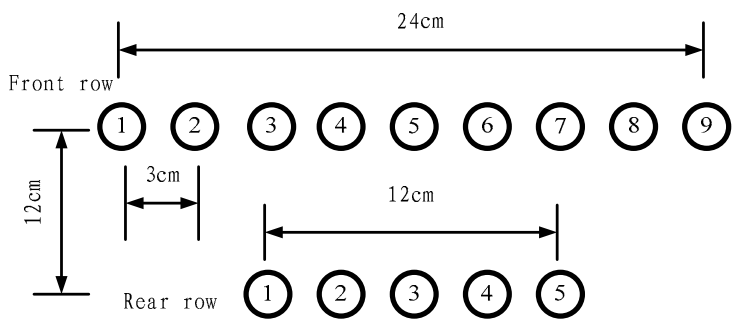

Figure 7. Layout of electromagnetic sensor coils

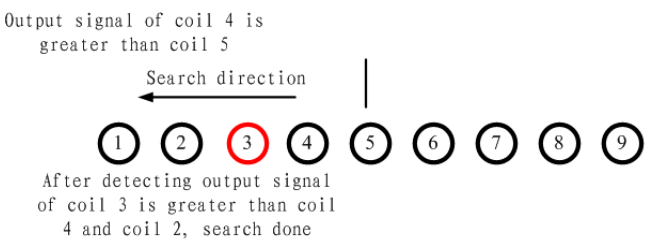

Figure 8. Searching method of output maximum signal coil

Conversely, if the output signal of coil 6 is greater than coil 5 , then using the same way mentioned above to find the maximum output signals coil from coil 7, 8 and 9 .

Using the same search method to find the coil of maximum output signal in rear row. This searching method could promote the searching efficiency, and the numbers of inductance coils are less than 7.

With the search method of maximum output signal coil, two coils could be selected in front and rear row, and they are the nearest ones to navigation wire.

Figure.9 shows the simulation running path of electromagnetic smart car, two black ovals represent the front wheel position of smart car; black solid curve represent the navigation wire, the straight dotted line through coil 5 in front row and coil 3 in rear row represent the axis of the smart car.
Using the searching method of maximum output signal coil, coil 3 in front row and coil 2 in rear row could be searched and are the nearest ones to navigation wire in Figure.9, so navigation wire could be approximated fitting to the dotted line through coil 3 in front row and coil 2 in rear row, the deflection angle of smart car steering equal the angle $\theta$ between the two dotted lines. Therefore the calculation formula for angle $\theta$ can be expressed as:

$$
\theta=\arctan \frac{|\mathrm{L} 3-\mathrm{L} 4|}{\mathrm{L}}
$$

In above formula, L3 represent the distance between coil 3 and coil 5 in front row, and it is $6 \mathrm{~cm} ; \mathrm{L} 4$ represent the distance between coil 4 and coil 5 in front row, and it is $3 \mathrm{~cm}$; $\mathrm{L}$ represent the distance between front row and rear row, and it is $12 \mathrm{~cm}$. In actually, establishing the relationship table between the value of $\tan \theta$ and PWM value of steering deflection angle replaces calculating the value of angle $\theta$ to control the direction and speed of the smart car.

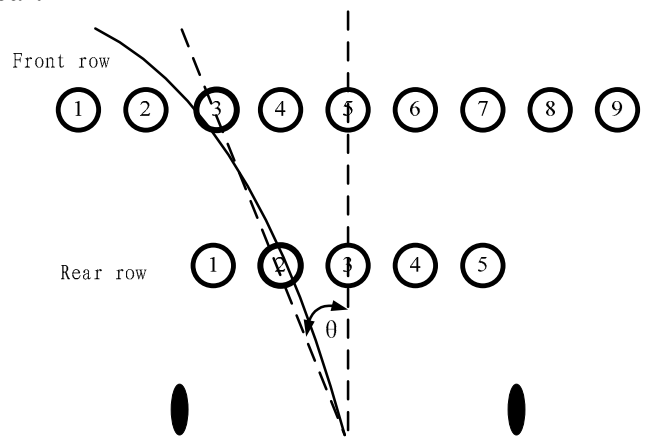

Figure 9. Running simulation chart of smart car

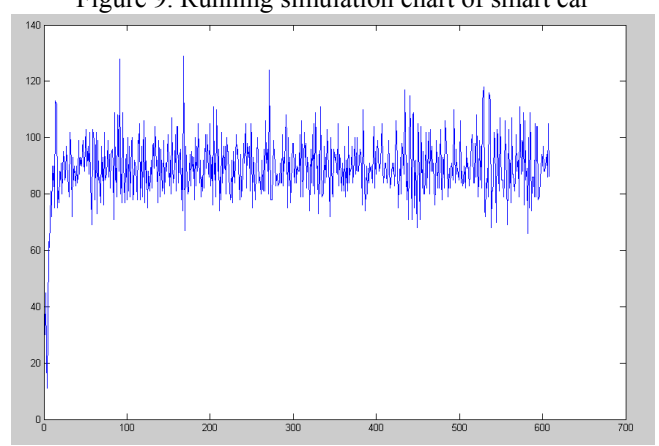

Figure 10. PID speed adjusting simulation chart

\section{SPEED CONTROL ALGORITHM}

The main control information of car's motor and steering comes from electromagnetic sensor module. General control principle is , the larger the $\tan \theta$, the greater the steering deflection angle, and the slower the smart car speed. Speed of smart car controlled by PID algorithm controller. Through the wireless transmission module, collect feedback speed datum for a period of time on straight path, these datum are transformed into dimensionless quantity, using MATLAB's plot function, PID algorithm simulation graph of smart car was shown in Figure.10. 
For straight and all kinds of curved navigation wire, the values of given speed vary, and PID algorithm adjustable parameters also vary. This kind of control strategy could make smart car running at its optimal speed, ensure smart car's stability running, and bring full speed potential into play

\section{SUMMARIES}

Through tests, electromagnetic smart car based on position detection method have good forward-looking, good stability and sensitivity, avoid control difficult of variation value of $\mathrm{AC}$ through navigation wire.
Electromagnetic smart car technology covers automation control, electromagnetic, sensor, electronics, computer and other relative areas, and can be applied to intelligent robots, industrial control, and transportation and so on. It is worthy to extend.

\section{REFERENCES}

[1] Competition Secretariat $.20 \mathrm{kHz}$ Power Supply Reference Design. Fifth National Student Smart Car Race, 2009,PP:1-5

[2] Competition Secretariat. Competition Cars Path Detection Reference Scheme of Electromagnetic Group. Fifth National Student Smart Car Race, 2010,PP: 1-8 